\title{
The Minimum Interval for Confident Spike Sorting: A Sequential Decision Method
}

\author{
Paul Hebert ${ }^{\dagger}$, Student Member, IEEE and Joel Burdick ${ }^{\dagger}$, Member, IEEE
}

\begin{abstract}
This paper develops a method to determine the minimum duration interval which ensures that the process of "sorting" the extracellular action potentials recorded during that interval achieves a desired confidence level of accuracy. During the recording process, a sequential decision theory approach continually evaluates a variant of the likelihood ratio test using the model evidence of the sorting/clustering hypotheses. The test is compared against a threshold which encodes a desired confidence level on the accuracy of the subsequent clustering procedure. When the threshold is exceeded, the clustering model with the highest model evidence is accepted. We first develop a testing procedure for a single recording interval, and then extend the method to multi-interval recording by using both Bayesian priors from previous recording intervals and recently developed cluster tracking procedure. Lastly, a more advanced tracker is implemented and initials results are presented. This later procedure is useful for real time applications such as brain machine interfaces and autonomous recording electrodes. We test our theory on recordings from Macaque parietal cortex, showing that the method does reach the desired confidence level.
\end{abstract}

\section{INTRODUCTION}

The process of "spike sorting," aims to separate the neuronal action potential waveforms recorded on a single extracellular electrode according to their different generating signal sources. Spike sorting is a crucial step in processing extracellular neural recordings not only for basic scientific research, but also for brain machine interfaces (BMIs). In a typical BMI architecture, action potentials are recorded from one or more electrodes over a short period of time. After action potentials are detected in this interval and then sorted, information extracted from the sorted action potentials is used to activate the BMI. Clearly, mistakes in the spike sorting process may lead to subsequent errors in scientific, clinical, or real-time interpretations of the recordings.

Because spike sorting is central to so many applications, there is a well developed literature on various spike sorting methods (e.g., [1]-[5]). Many spike sorting methods use some variant of clustering to effect the separation of actions potentials. The detected action potential waveforms are projected onto a lower dimensional feature space, and the resulting projections are then grouped by a clustering procedure. Waveforms which fall into well defined groupings are then associated to a single generating source.

While spike sorting algorithms have reached a high level of maturity, a basic problem remains open. What is the

This work was supported by the National Institutes of Health (NIH)

${ }^{\dagger}$ Dept. of Mechanical Engineering, California Institute of Technology, Pasadena, CA 91125, USA paul.hebertecaltech.edu, jwberobotics.caltech.edu minimum duration of the recording interval that ensures an accurate clustering of the data in that interval? Conventionally, the recording process is halted at an a priori time, at which point clustering is performed on the recorded data. Two problems may occur. First, when the interval is too short, the data is too sparse to reliably cluster, and errors propagate into the subsequent application. Second, if the interval is too long, any decision based upon the clustered data will be delayed unnecessarily. Closed loop system performance can suffer due to such latencies. The critical problem then is to determine the termination time when the recording can be stopped in order to ensure a high confidence in the resulting sorting procedure. This paper develops a method, based on sequential decision theory, to determine the recording termination time which meets a predetermined level of confidence.

\section{Clustering BACKGROUND}

Many sorting algorithms are based on a clustering process, which generally proceeds as follows. An extracellular 1-D electrode voltage signal, $\mathcal{S}$, is recorded over an interval, $T$, of an as yet undetermined duration $\Delta$. The signal may be recorded over a single interval $T_{1}$, or the recording process may naturally be organized into successive intervals $T_{1}, T_{2}, \ldots, T_{F}$. The signal $\mathcal{S}$ is digitally sampled at interval $\delta$ so that $D \delta=\Delta$ for some integer $D$. Individual spike waveforms are detected (we use the method of Nenadic \& Burdick [6]) and isolated from $\mathcal{S}$. To reduce computational complexity, the detected spike waveforms $s_{i}$ (indexed by i) to be sorted are discretized equally and projected onto a 2-d Principal components axis (PCA). These projected waveforms $y_{i}$ form the clustering data. We briefly review two related clustering methods.

\section{A. Maximum Likelihood (ML) Clustering}

The classical ML clustering is a unsupervised method based on a Gaussian Mixture Model (GMM). A GMM models the data as if it were generated from a mixture of probability models (in this instance, a bivariate gaussian distribution), each representing a different cluster. Note, our method can work for any other clustering procedure for which the model evidence (see below) can be computed.

The cluster of action potential waveforms generated by the $g^{\text {th }}$ neuron is modeled as a Gaussian distribution $f_{\mathcal{N}}$. Let $Y=\left\{y_{i}\right\}_{i=1}^{N}$ denote the $N$ action potentials detection in $T$. Assuming independence of measurements, the likelihood of 
a given model $\mathcal{M}_{m}$ (a particular clustering of the data) is

$$
p\left(Y \mid \Theta, \mathcal{M}_{m}\right)=\prod_{i=1}^{N} \sum_{g=1}^{G} \pi_{g} f_{\mathcal{N}}\left(y_{i} \mid \mu_{g}, \Sigma_{g}\right),
$$

where $\mu_{g}, \Sigma_{g}$, and $\pi_{g}$ are the mean, covariance, and mixture weights, respectively of the $g^{\text {th }}$ cluster $g=1, \ldots, G$ (where the number of clusters $G$ in $T$ is not necessarily known). Classically, the number of clusters, and the assignments of each data point to a cluster (from which the cluster mean and covariance are determined) are estimated using Expectation-Maximization (EM) applied to (1), followed by model selection.

\section{B. Maximum a Prior (MAP) Clustering}

Wolf and Burdick [7], extended the classical approach to optimization of the posterior so that clustering results from a previous recording intervals can be used as a prior:

$$
p\left(\Theta_{k} \mid Y^{1: k}\right) \propto p\left(Y^{k} \mid \Theta_{k}\right) p\left(\Theta_{k} \mid Y^{1: k-1}\right),
$$

where $k$ indexes the clustering time interval and $Y^{1: k}=$ $\left\{Y^{1}, \ldots, Y^{k}\right\}$ denotes the collection of projected waveforms found in intervals $T_{1}$ to $T_{k}$. Because a given neuron's cluster mean varies slowly over time, while the other model parameters $\Sigma_{g}^{k}$ and $\pi_{g, k}$ vary greatly from one interval to another, the prior is chosen as a mixture of the previously found cluster centers

$$
p\left(\Theta_{k} \mid Y^{1: k-1}, \mathcal{M}_{m}\right)=\prod_{g=1}^{G} \sum_{j=1}^{\hat{G}_{k-1}} \frac{1}{\hat{G}_{k-1}} f_{\mathcal{N}}\left(\mu_{g, k} \mid \psi_{j}^{k}\right),
$$

where $\hat{G}_{k-1}$ is the estimated number of clusters in $T_{k-1}$ and $\psi$ is comprised of the estimated mean center $\hat{\mu}_{j, k-1}$ and the covariance, $S_{j, k \mid k-1}$, associated with that estimate. This MAP approach not only associates spikes to generating neurons, but also associates neuron clusters over time to effect "tracking" of individual neurons. While, this approach does not account for possible appearance and disappearance of clusters, we have also successfully applied this method to a more complex cluster-tracker method which account for such events [12].

\section{Sequential Decision Theory}

The central goal of this paper is to find a statistical test which can determine the time, $\Delta_{k}$, at which we have sampled a sufficient amount of data to cluster and proceed with any subsequent decision where reliability of the clustering process is important. The sequential probability ratio test (SPRT) [8] provides a statistical hypothesis testing framework for sequentially sampled data. It allows for quicker decisions than classical hypothesis testing, which draw conclusions based upon a predetermined number of sample points. Under appropriate conditions, the SPRT reaches a decision with the minimum number of samples, thereby providing the shortest termination time [8]. It incorporates a ratio of two joint probability densities - each with density parameters $\theta$. Let $f(x, \theta)$ denote the distribution of random variable $x$ with parameters $\theta$. Let $H_{1}$ be the hypothesis that $\theta=\theta_{1}$ (so that the distribution of $x$ is $f\left(x, \theta_{1}\right)$ ) and $H_{0}$ be the hypothesis that $\theta=\theta_{0}\left(f\left(x, \theta_{0}\right)\right.$ holds when $H_{0}$ is true $)$. For each successive data sample $x_{n}(n=1, \ldots, N)$, the SPRT computes the following test:

$B<\frac{p_{1 N}}{p_{0 N}} \equiv \frac{p\left(H_{1} \mid x_{1}, \ldots, x_{N}\right)}{p\left(H_{0} \mid x_{1}, \ldots, x_{N}\right)}=\frac{f\left(x_{1}, \theta_{1}\right) \ldots f\left(x_{N}, \theta_{1}\right)}{f\left(x_{1}, \theta_{0}\right) \ldots f\left(x_{N}, \theta_{0}\right)}<A$

where A and B represent the stopping rules for the data collection. Wald approximated these rules as:

$$
A=\frac{1-\beta}{\alpha}, \quad B=\frac{\beta}{1-\alpha},
$$

where $\alpha$ is the probability of committing an Type I error (accepting hypothesis $H_{1}$ when $H_{0}$ is true) and $\beta$ is the the probability of committing a Type II error (accepting $H_{0}$ when $H_{1}$ is true). If the ratio is greater than $A$, we conclude that hypothesis $H_{1}$ is true. If the ratio is less than $B$, we conclude $H_{0}$ is true. Else, an additional sample is taken, the ratio is recalculated, and the decision is evaluated again. This test will terminate with probability of 1 [8].

Armitage [9] extended the SPRT to the case of multiple hypotheses, which is needed for the spike sorting problems considered in this paper. For $L$ different clustering hypotheses, the probability density ratios of different hypothesis combinations are evaluated. Let $R_{n l}$ denote a probability density ratio (evaluated at a sample point $x_{n}$ ) between hypothesis $H_{l}(l=1, \ldots, L-1)$ and hypothesis $H_{L}$ :

$$
R_{n l}=\frac{f\left(x_{n}, \theta_{l}\right)}{f\left(x_{n}, \theta_{L}\right)} .
$$

The multi-hypothesis SPRT (MSPRT) for $L$ hypotheses (evaluated at the $n^{\text {th }}$ data sample) can then be expressed as:

$$
\left.\begin{array}{c}
\sum_{n=1}^{N}\left(\log R_{n l}-\log R_{n p}\right)>\mathcal{T} \quad \forall p \neq l \\
\sum_{n=1}^{N}\left(\log R_{n l}\right)>\mathcal{T}
\end{array}\right\} \text { Accept } H_{l}(l \neq L)
$$

or

$$
\left.\sum_{n=1}^{N}\left(\log R_{n p}\right)<-\mathcal{T} \quad \forall p=1, \ldots, L-1\right\} \text { Accept } H_{L}
$$

I.e., all comparisons of $H_{l}$ to every remaining hypothesis must exceed a stopping rule for $H_{l}$ to be accepted. Note the upper stopping rules are chosen equal to eliminate any bias towards a particular hypothesis. Armitage developed a conservative threshold as

$$
\mathcal{T}=\log \left(\frac{L-1}{1-\mathcal{P}_{i i}}\right)
$$

where $\mathcal{P}_{i i}$ is the probability of choosing $H_{i}$ when $H_{i}$ is true. As in classical SPRT testing, if neither of the above tests pass their corresponding thresholds, the sampling procedure continues until the test terminates.

The MSPRT theory can be applied in two ways to the recording interval termination problem. First, it can naturally 
be applied to a single recording interval. In theory, the MSPRT could be evaluated as frequently as the arrival of every new spike waveform. Practically, the MSPRT will be evaluated a frequent intervals (e.g., every 0.5 seconds) within the overall recording interval $T$. Second, as needed for real-time BMI applications which rely upon short sequential recording intervals, the MSPRT can determine the shortest interval based on only the recordings in that interval, but also incorporating prior clustering results from previous intervals.

\section{MSPRT BASEd UPON THE MOdEL EVIDENCE}

Since multiple models can often plausibly explain a given neural data set, the adoption of MSPRT to this problem is ideal. Implementing MSPRT requires a probability that measures the validity of each clustering hypothesis. For this, we propose the model evidence, $p\left(Y^{k} \mid \mathcal{M}_{m}\right)$, as the measure in (5), as it assesses how well the data conforms to the given model $\mathcal{M}_{m}$. As sequential samples are clustered, the evidence for the best model will become more pronounced. When the leading model's evidence surpasses that of all other models by a threshold, calculated by (6), the termination time is found.

Popular approaches to calculating or approximating the model evidence are the Akaike Information Criterion (AIC) [10] and the Bayesian Information Criterion (BIC) [11]. However, these two criterion do not incorporate prior clustering results. Nonetheless, we have examined the use of the BIC with the sequential decision theory:

$$
B I C \equiv-2 \log l_{M}\left(\hat{\Theta}_{m}^{k} \mid Y^{k}, \mathcal{M}_{m}\right)+\eta_{m} \log N .
$$

For the case without prior information, (7) can be used for the probability expression in (5).

In order to incorporate prior knowledge, Wolf [12] estimated the model evidence, $p\left(Y^{k} \mid Y^{1: k-1}, \mathcal{M}_{m}\right)$ using Laplace's method as an approximation to the marginalizing integrals:

$$
\begin{aligned}
& p\left(Y^{k} \mid Y^{1: k-1}, \mathcal{M}_{m}\right) \approx p\left(Y^{k} \mid \hat{\Theta}_{m}^{k}, \mathcal{M}_{m}\right) \\
& \quad p\left(\hat{\Theta}_{m}^{k} \mid Y^{1: k-1}, \mathcal{M}_{m}\right)(2 \pi)^{\eta_{m} / 2}\left|\mathbf{H}\left(\hat{\Theta}_{m}^{k}\right)\right|^{-1 / 2}
\end{aligned}
$$

where $\eta_{m}$ is the number of independent parameters in model $\mathcal{M}_{m}$. The first term is the mixture likelihood expressed in equation (1) and the second term is the prior (2). The Hessian matrix, $\mathbf{H}$, is the second gradient with respect to the parameters $\Theta_{k}$ of the first two terms.

We also use the model class probability as a supporting decision rule to prevent a premature decision which may occur when few data points are yet recorded. In this case, the clustering process may clump all of the data points into a single cluster, which often produces a high model evidence. In such cases, the model class probability will be low, thereby preventing a premature termination. The model class probability can be found by Bayes' rule:

$$
P\left(\mathcal{M}_{m} \mid Y^{1: k}\right)=\frac{1}{\mathrm{C}} \underbrace{p\left(Y^{k} \mid Y^{1: k-1}, \mathcal{M}_{m}\right)}_{\text {model evidence }} \underbrace{P\left(\mathcal{M}_{m} \mid Y^{1: k-1}\right)}_{\text {model prior }},
$$

where $\mathrm{C}$ is a normalizing constant. In our experiments, MSPRT was applied to both the model evidence and the model class probability. Only when both tests pass is the termination time accepted.

\section{EXPERIMENTAL RESULTS}

Data obtained in acute single electrode recording sessions from the parietal cortex of a macaque monkey carrying out a center-out reaching task were used to evaluate our result (this data and recording method was published in [12]). Electrode signals were sampled at a rate of 10,000 Hz. Spikes were detected using the method of [6], and the extracted waveforms were projected to a 2-dimensional PCA space, where the clustering methodology of Section II-B was applied.

We applied the sequential decision theory from Section IV to a sequence of 12 consecutive recording intervals ( $k=$ $1,2, \ldots, 12)$ whose duration was arbitrarily set to 10 seconds during the initial recording sessions. We sought to determine if a clustering decision could have been reached earlier than the pre-set 10 second termination. While the termination decision could have been evaluated for every successive spike, to simulate practical applications, the MSPRT test was evaluated after every 1 second of recording. Wolf's method was used to determine the model evidence.

Using the model-evidence-based test (ME-MSPRT) of Section IV, with a certainty level of $\mathcal{P}_{i i}=0.9$, our method predicted a confident clustering in 5 out of the 12 intervals before the 10 second terminus. The intervals that passed both tests had error rates well within the prescribed certainty level, as determined with a laborious hand-sorting of the data. The error is calculated as the mean of the cluster's errors

$$
\text { Error }=\frac{1}{G} \sum_{g=1}^{G} \frac{\mathrm{MC}_{g}+\mathrm{FC}_{g}}{n_{g, \text { truth }}},
$$

where MC is the number of missed clusterings (a spike feature belonging to $g^{\text {th }}$ cluster is not correct assigned) and FC is the number of false clusterings (a spike is mis-assigned to the $g^{\text {th }}$ cluster) and $n_{g, \text { truth }}$ is the ground-truth number of spikes within the $g^{\text {th }}$ cluster.

The method was then applied to a larger data set consisting of 150 ten second recording intervals gathered during a single 3 hour recording session. Fig. 1a displays the clustering error of those 79 intervals in which the termination time was found to be less than 10 seconds using the ME-MSPRT. Note that only 6 out of 79 recording intervals had clustering errors of more than $10 \%$. Thus, ME-MSPRT had a $2.1 \%$ mean error rate given the certainty level of $\mathcal{P}_{i i}=0.9$.

Fig. 1b shows the influence of the certainty level. The ME-MSPRT test was applied to the same 150 recording intervals but each with a different certainty level. Naturally as one would expect, as the certainty level increases, the mean clustering error decreases. Even with a low certainty level of $\mathcal{P}_{i i}=0.75$, the clustering error was still less than $5 \%$. This was expected as the thresholds in (4) and (6) are considerably conservative [8], [9]. Only at $\mathcal{P}_{i i}=0.99$ did the test break down providing an error of only $1.82 \%$. 
A reasonable explanation is that at high confidence, more data must be analyzed to improve the error estimate as considerably less intervals pass the test.

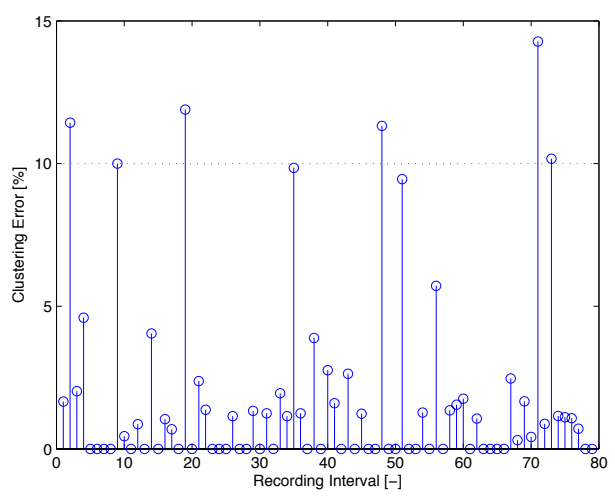

(a) ME-MSPRT for $\mathcal{P}_{i i}=0.9$

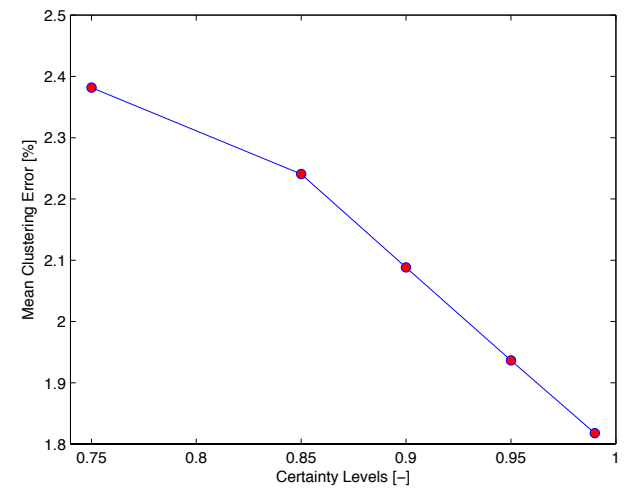

(b) ME-MSPRT Trend

Fig. 1: Clustering errors for $<10 \mathrm{sec}$. terminated intervals

We also implemented a more complex tracker and test with the adoption of multiple hypothesis tracking for clusters (MHTC). Details of MHTC may be found in [12]. The test (MHTC-MSPRT) based on the Global Hypothesis Probability for (5) was implemented on the same data set. Fig. 2a displays 134 intervals now in which the termination time was found to be less than 10 seconds, considerably more than with ME-MSPRT. MHTC-MSPRT provides a $69.6 \%$ improvement and is better in determining the shortest reliable interval for clustering. Note that only 2 out of 134 of these recording intervals had clustering errors of more than $10 \%$. Thus, MHTC-MSPRT had a $1.9 \%$ mean error rate given the certainty level of $\mathcal{P}_{i i}=0.9$.

\section{CONCLUSion ANd Future Work}

The required duration for a recording interval to provide reliable spike sorting/clustering is a practical, but heretofore an unsolved problem. The adoption of sequential decision theory into a clustering framework allows for the determination of the reliable termination time with respect to a predetermined user-defined confidence level. This framework prevents errors from propagating into clinical analysis when

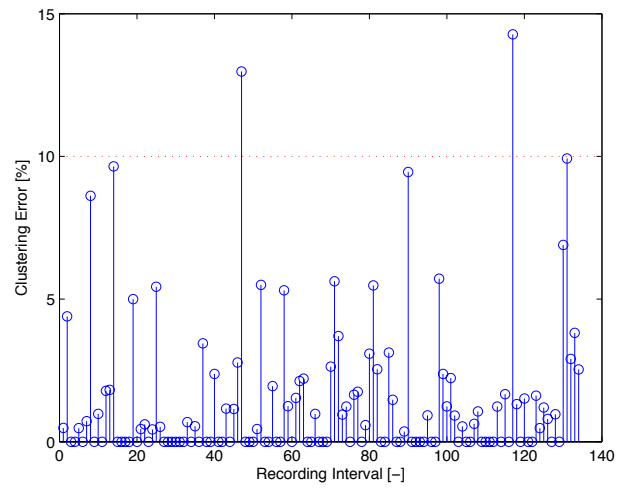

(a) MHTC-MSPRT for $\mathcal{P}_{i i}=0.9$

Fig. 2: Clustering errors for $<10 \mathrm{sec}$. terminated intervals

data is sparse or the interval too short, while minimizing delays in real-time applications. The adoption of a more advanced tracker into our framework provides better performance in clustering error and shorter minimum intervals for reliable clustering. Our experiments show that mean clustering errors are less than the specified confidence, except in cases of extreme desired reliability (99\%), where finite sample issues hamper the analysis.

Acknowledgements: The authors thank Prof. Richard Andersen, Dr. Michael Campos, and Dr. Michael Wolf for providing the neural data sets.

\section{REFERENCES}

[1] M. S. Lewicki, "A review of methods for spike sorting: the detection and classification of neural action potentials," Network: Computation in Neural Systems, vol. 9, pp. R53-R78, 1998.

[2] — "Bayesian modeling and classification of neural signals," Neural Computation, vol. 6, no. 5, pp. 1005-1030, 1994.

[3] S. Shoham, M. R. Fellows, and R. A. Normann, "Robust, automatic spike sorting using mixtures of multivariate t-distributions," Journal of Neuroscience Methods, vol. 127, no. 2, pp. 111 - 122, 2003.

[4] R. Q. Quiroga, Z. Nadasdy, and Y. Ben-Shaul, "Unsupervised spike detection and sorting with wavelets and superparamagnetic clustering," Neural Computation, vol. 16, no. 8, pp. 1661-1687, 2004.

[5] E. Wood, M. Fellows, J. Donoghue, and M. Black, "Automatic spike sorting for neural decoding," in Engineering in Medicine and Biology Society, 2004. IEMBS '04. 26th Annual International Conference of the IEEE, vol. 2, Sept. 2004, pp. 4009-4012.

[6] Z. Nenadic and J. W. Burdick, "Spike detection using the continuous wavelet transform," Biomedical Engineering, IEEE Transactions on, vol. 52, no. 1, pp. 74-87, Jan. 2005.

[7] M. T. Wolf and J. W. Burdick, "Bayesian clustering and tracking of neuronal signals for autonomous neural interfaces," in Decision and Control, 2008. CDC 2008. 47th IEEE Conference on, Dec. 2008, pp. 1992-1999.

[8] A. Wald, Sequential Analysis. New York; London: J. Wiley \& Sons; Chapman \& Hall, 1947.

[9] P. Armitage, "Sequential analysis with more than two alternative hypotheses, and its relation to discriminant function analysis," Journal of the Royal Statistical Society, vol. 12, no. 1, pp. 137-144, 1950.

[10] H. Akaike, "A new look at the statistical model identification," Automatic Control, IEEE Transactions on, vol. 19, no. 6, pp. 716723, Dec 1974.

[11] G. Schwarz, "Estimating the dimension of a model," The Annals of Statistics, vol. 6, no. 2, pp. 461-464, 1978.

[12] M. T. Wolf, "Target tracking using clustered measurements, with applications to autonomous brain-machine interfaces," $\mathrm{Ph}$.D. dissertation, California Institute of Technology, May 2008. 